\title{
Modelling Cepheids and RR Lyrae Stars
}

\author{
G. Kovács \\ University of Florida, Department of Physics, Gainesville \\ and \\ Konkoly Observatory, Budapest, Hungary
}

\begin{abstract}
We review the recent nonlinear hydrodynamical results and the numerical problems to be solved by the next generation of codes. The behavior of the Fourier parameters and double-mode RR Lyrae pulsation will be discussed in detail. We emphasize the importance of the implementation of the various adaptive-mesh schemes for stellar pulsations. A further goal is the substitution of the artificial viscosity with modern shock capturing methods and a better treatment of radiative transfer. Convection however, must ultimately be included in order to supply the necessary physical mechanism for dissipation.
\end{abstract}

\section{Introduction}

The application of the full set of nonlinear equations of radiative hydrodynamics to the modelling large-amplitude stellar pulsations has a history of almost 30 years. Since the pioneering work of Christy (1964) there have been a number of attempts to improve the original idea. The particular problems to tackle in nonlinear stellar pulsation are as follows: (1) there is a very steep temperature and density gradient at the hydrogen ionization zone which moves according to the local ionization conditions, therefore, does not follow the motion of the mass elements; (2) under certain conditions shocks may develop which also move according to the local thermo- and hydrodynamical situation; (3) the exact solution of the radiative transfer problem coupled with the hydrodynamics is very difficult throughout the entire stellar envelope and atmosphere, because of the basic change of the radiative properties in these regions; (4) convection should play a role in the pulsation, which poses the Herculean task of simulating the three-dimensional turbulent flow by a one-dimensional model; (5) since we are interested in the long-term behavior, the code should be very stable numerically and exact total energy conservation is a must. Past code developments handled some of these questions with some success. For reference we mention Davis (1972), Stellingwerf (1974, 1975, 1984), Castor, Davis and Davidson (1977), Aikawa and Simon (1983), Bowen (1988). Since, and parallel with these works, there have been a lot of progress made in solving one- and multi-dimensional hydrodynamical problems in various fields of physics and engineering. A realization of this fact stimulated the organization of a recent workshop on this topic (Buchler 1990).

The purpose of this paper is threefold: (i) to review the major recent results and problems in modelling Cepheid and RR Lyrae pulsations; (ii) to emphasize the 
importance and present a possible mechanism for multimode stellar pulsation among classical variables; (iii) to give a flavour of the latest efforts in code developments.

\section{Stellar masses with the new opacities}

During the last two years the widely used Los Alamos opacities have undergone substantial changes following the bold suggestion of Simon (1982). The OPAL opacities of Rogers and Iglesias (1992) cured the basic discrepancy of the beat Cepheids (Moskalik, Buchler and Marom 1991). A similar, but maybe less accommodating conclusion can be drawn by the application of the OP opacities (Seaton; Kanbur, these proceedings). For bump Cepheids, on the other hand, the opacity enhancement did not prove to be completely sufficient (Moskalik et al. 1991). On the other hand, a subsequent improvement of the OPAL opacities with the consideration of the spin-orbit interaction (Iglesias, Rogers and Wilson 1992) further decreased (in our opinion eliminated) the difference between bump and evolutionary masses (Moskalik, these proceedings).

While solving the Cepheid mass discrepancies, the OPAL opacities led to an unexpected sensitivity to metal abundance and chemical composition, and confused the situation of RRd stars (Kovács, Buchler and Marom 1992; Kovács et al. 1992). Considering only luminosity and iron abundance effects, for solar composition the ranges of RRd masses are $0.75-0.8$ and $0.7-0.9 M_{\odot}$ for M15 (Oo II) and IC 4499 (Oo I) respectively. By taking the 'standard' values of the luminosities, iron abundances and individual periods, there seems to be no systematic difference in mass between the two Oosterhoff type RRd stars. The ambiguities in the observed chemical composition and metal abundances, however, do not permit us to draw any firm conclusion yet.

\section{Fourier decomposition}

\section{3a. Bump Cepheids}

One important utilization of nonlinear hydrodynamics is to compare the theoretical pulsation profiles with the observed ones. A natural frame of reference is the set of Fourier parameters obtained from a fit to the pulsation cycle. Before considering the ultimate goal of fitting the light and velocity curves of the individual stars we attempt to verify the overall trend in the progression of the Fourier parameters.

Because of the new opacities, the center of the $2 \omega_{0}=\omega_{2}$ resonance for bump Cepheids is located at 10 days, therefore, the hydrodynamical results can be compared directly with the observations. Such a comparison shows that the overall agreement with the observations is very good, at least for the Fourier parameters up to the third order (Moskalik et al. 1991). There are, however, some systematic differences, especially in $\phi_{21}$ for the light curves of the low-period stars. Further characteristic features which have not yet been modelled, are the break in the $\phi_{31}$ and $\phi_{41}$ progression at $\approx 7$ days for the light curves and the strict linear relation between $\phi_{31}$ and $\phi_{41}$ after 10 days (Simon and Moffett 1985). There is a possibility that the feature at 7 days is associated with the $3 \omega_{0}=\omega_{4}$ resonance (Antonello, these proceedings). 
Clearly, understanding these characteristics is an important task for future hydrodynamical simulations. Further detailed discussions of the bump Cepheid progression are in Buchler, Moskalik and Kovács (1990) and Moskalik et al. (1991) and references therein.

\section{3b. s-Cepheids}

Using the Fourier decomposition technique, Antonello and Poretti (1986) found that a large fraction of the small amplitude Cepheids below $\approx 5$ days follow a progression in their Fourier parameters different from that of the Classical Cepheids. Because of their sinusoidal light curves and low amplitudes, a common understanding is that s-Cepheids are first overtone variables. An apparent break in their $\phi_{21}$ could then be accounted for by a $2 \omega_{1}=\omega_{4}$ resonance, very similarly to that of the bump Cepheids. Unfortunately, there is very little direct observational evidence so far that s-Cepheids are indeed first overtone pulsators. Though nonlinear results suggest something similar to that what we observe, they are not convincing either. We refer to Antonello's paper of these proceedings for the present status of these variables. It is clear that a more detailed study of the SMC first overtone Cepheids would help to clarify the situation of the s-Cepheids (Smith et al. , these proceedings).

\section{3c. RR Lyrae stars}

Unlike Classical Cepheids, RRab stars do not show any systematics in the variation of their Fourier parameters as a function of the period (Simon and Teays 1982). Similarly to the bump Cepheids, there are systematic differences in the low-order Fourier phases (Simon 1985). Our unpublished tests confirmed this discrepancy basically independently of the opacity and artificial viscosity (see also Kovács 1990). The obvious agents to resolve these discrepancies are convection and more accurate treatment of radiative transfer. Both of these were absent from the codes used in the above computations.

RRc stars, on the other hand, seem to follow some trend in their Fourier parameters as a function of the period. Also, nonlinear models are in a fair agreement with the observations. The roughly linear relation between the period and $\phi_{31}$ prompted Simon (1989) to use this phase as a diagnostic tool to obtain stellar parameters. Further discussions of the RRc stars in this context are given by Simon and by Cacciari and Bruzzi, these proceedings.

\section{Mode selection, double-mode pulsation}

One of the main goal in modelling nonlinear stellar pulsation is the determination of the long-term behavior of the model. In this respect the application of the relaxation technique (Stellingwerf 1974) to find strictly periodic solutions and their stability is extremely important. In the case of coexisting stable single- and multimode, or unstable single-mode solutions however, the direct (i.e. 'brute force') time integration cannot be avoided. In this situation the final state can be assessed only by 'long enough' integration combined with a precise analysis of the time-series. 
A further major complication in the mode selection is the important role of various viscosity parameters (artificial and eddy). There is probably an optimal tradeoff in the amount of viscosity in order to get a result which is not very sensitive to these parameters, but at the same time to maintain the numerical stability and observationally acceptable amplitude. Until further improvement in the shock treatment and convection theory, statements about mode selection remain somewhat uncertain, especially close to the mode transition (i.e. at the bifurcation).

Modelling double-mode pulsation has been a serious challenge for nonlinear hydrodynamics for decades. Here we would like to give only the highlights of the very encouraging developments of this year. A more comprehensive review and details of our double-mode models are given in Kovács (1992); Kovács and Buchler (1992).

The most important constraints for any model compatible with the observations are : (a) periods, (b) amplitudes, (c) amplitude stability. Although previous modelling has already produced double-mode RR Lyrae pulsation in the vicinity of the $2 \omega_{0}=\omega_{3}$ resonance (Kovács and Buchler 1988), the requirement of matching the observed periods was not met. Since no low-order resonance could fit the period and period ratio at the same time, we tried to approach the question from another angle. As is well known, viscosity plays an important role in some circumstances in controlling mode selection. To avoid this, we started a survey of low dissipative RR Lyrae models in 1991. The basic question we would like to answer is: how would a purely radiative $R R$ Lyrae model pulsate if we could get rid of all the unnecessary dissipation? These models have higher amplitudes (especially velocities) than those given by the observations, but we defer this question to a later time (see also Section 6). Quite surprisingly, but the low-viscosity models showed a window of unstable fundamental pulsation, which for somewhat cooler models overlapped with the 'fundamental only' region and resulted in a double-mode pulsation.

Many models have been thoroughly studied by limit cycle analysis and by very long direct time integrations. New features of pulsation were detected, like periodically varying mode amplitudes and coexisting double- and single-mode states. Though the periods were matched and the double-mode state was steady, the amplitude ratios were reversed compared to what is observed. It turned out however, that this problem can be cured by allowing the models to be a little more dissipative. The higher dissipation led to the generation of another double-mode state, now with the right, directly observable quantities.

It is, of course, very important to confirm and further refine our finding by independent computations which use different numerical schemes and perhaps more complete physics (most importantly convection). Marvelously enough, it was at this conference that such a supporting new results were presented for the first time. Though at different stellar parameters, new convective models of Bono and Stellingwerf show the same type of double-mode behavior as our radiative minimal viscosity models. It is remarkable too that their double-mode model also appears at the neighbourhood of the same type of resonance (namely $3 \omega_{0}-\omega_{1}=\omega_{2}$ ) what we have claimed to be responsible for the double-mode pulsations in our models. 
An even more recent development is a test of one of our double-mode model with a new radiative adaptive code (Marom, private communication). The model settled down with the new code to a very similar double-mode state found by our Lagrangean code.

Though it is clear that further work is necessary on the convective models to get better agreement with the observations, we think that this first result is very encouraging. By using updated opacities and extending the survey to a larger parameter space, convective models might cure the problem of low luminosity and temperature of our radiative models.

\section{Stochastic effects in classical variables}

There is no star in the classical instability strip which avoids convection. As a consequence, turbulence (i.e. stochastic forcing) is a natural ingredient of pulsation. Convective pulsation models usually take into consideration that part of the stochastic effect which have nonzero ensemble average (e.g. pressure, flux). The remaining fluctuating part has so far been neglected in the studies of the classical variables, because of the assumed small effect of the random perturbations which cancel out on the average. As is well known, this part of the turbulent convection plays an important role in the theory of the stochastic mode excitation of the 5 min. solar oscillations (Goldreich and Keeley 1977). In that theory the overall linear damping and the turbulent intensity which excite the modes balance each other, because both depend mostly on the same physical mechanism, i.e. turbulent convection. We would like to indicate here that in the case of limit cycle pulsation the situation is quite different, which may lead to much higher amplitudes of the stochastically excited modes.

Let us consider a single periodic pulsation close to the transition region. Because the pulsation is stable, all modes are damped in the limit cycle. Usually, as in the case of non-resonant pulsation, the linearly damped modes are even more damped in the limit cycle. The linearly excited modes, however, are arbitrarily mildly damped, and at the transitions they become actually excited with a rate which is less than their linear excitation rates. There are two possibilities to consider: (a) the model is in the 'one limit cycle only' region, or (b) the model is in the 'either-or' region. In case (a) the modes can reach in principle arbitrarily large amplitudes depending on the intensity of the noise. In case (b), however, there is an upper limit on the amplitudes of the noise generated (precursor) modes, because increasing the noise causes not only an amplitude increase, but also the chance that the model switches to the other, more stable limit cycle. Both situations are generic, but in the stellar situation we consider case (b) more likely to lead to observable precursor oscillations, because in principle, close to the transition arbitrary small noise may lead to observable amplitudes. Work is in progress to estimate the mode amplitudes and lifetimes.

An important question is the intensity of the noise. An ab initio estimation of this quantity is very complicated and risky (Goldreich and Keeley 1977; Goldreich and Kumar 1990). From the observation of the long-term phase variation, however, one 
may get some direct estimation on the size of the noise. Very often cited features of the O-C diagrams are the irregular and 'faster than the evolutionary time scale' variations. We think that at least a part of these problems can be explained by stochastic phase diffusion, which has basically a non-stationary, random walk character.

We think that a search for low-amplitude pulsation in classical variables would be extremely useful. If such a search were positive, we could map the period ratio diagrams or help to estimate masses and chemical compositions much more accurately than the RRd stars allow (see Kovács and Buchler, these proceedings).

For further discussion of the above topic, the general formalism and some interesting dynamical effects of noise, we refer to Kovács and Buchler (in preparation); Buchler, Goupil and Kovács (1992); and Buchler and Kovács (1992).

\section{The new generation of pulsation codes}

The basic numerical technique of nonlinear stellar pulsation is more than 20 years old (Fraley 1968). Though together with Stellingwerf's (1974) relaxation method the scheme is a very powerful tool in studying nonlinear stellar pulsation, obvious improvements are necessary. We emphasize here the importance of the further sophistication of the radiative hydrodynamics. For the problem of convection we refer to Stellingwerf (these proceedings).

As it was mentioned in Section 1, the main problems to be solved in the radiative nonlinear codes are: (i) proper tracing of the hydrogen ionization zone; (ii) substituting the artificial viscosity method by some modern, less dissipative shock capturing scheme; (iii) more accurate solution of the radiative transfer problem throughout the entire envelope.

In the last two years some progress has been made in tackling most importantly, the tracing of the hydrogen ionization. Problems (ii) and (iii) have not yet received proper attention perhaps of the possibly greater importance of problem (i) (see however Buchler and Whalen 1990; Dorfi and Feuchtinger 1991; Gehmeyr 1991).

The first adaptive pulsation code was constructed by Castor et al. (1977). Aiming specifically to resolve the hydrogen ionization zone, Aikawa and Simon (1983) opted for a scheme in which the updated temperature plays the role of the independent spatial variable. The new scheme of Gehmeyr (1991) is in principle able to resolve any feature specified in the structure function. Another new technique was suggested by Dorfi and Gautschy (1990) and later implemented by Dorfi and Feuchtinger (1991). In all these works the total energy conservation, a very important quality of nonlinear pulsation, was not guaranteed by the schemes. In addition, in the limit of no spatially resolved structures, the above schemes go to an Eulerian coordinate system instead of a Lagrangean one, which is a more natural system for a radially pulsating star. The new adaptive code developed by Buchler and Marom (1992) has this additional useful feature and furthermore, exactly conserves the total energy.

Beside the very smooth variation of the physical quantities, adaptive codes, in general, also seem to give smaller amplitudes than those of the Lagrangean codes (see however Gehmeyr 1991). Radial velocities usually decrease more than luminosity 
amplitudes (Kovács 1990; Marom, private communication). Together with the more accurate solution of the hydrodynamical equations, the amplitude decrease and the smooth variation shows that adaptive codes provide a more sound modelling of the pulsation and may even suggest that (at least at the hotter side of the instability strip) convection might be not all that important dynamically.

\section{Conclusion}

Substantial progress has been made in modelling classical stellar pulsations during the last two years. The revised opacities have led to the elimination of the frustrating Cepheid mass discrepancies. New thorough survey of radiative RR Lyrae models with minimal viscosity has produced the first physically sound double-mode models, thereby breaking the dead-lock of search for RRd models and stimulating further studies in this field. A new generation of nonlinear pulsation codes are under development, which aim it is to solve the complex problem of radiative hydrodynamics very accurately.

We hope that these developments will ultimately lead to a more fruitful application of nonlinear pulsation results, like fitting the theoretical pulsation profiles to the observations of the individual stars. Understanding mode selection, multimode behavior and long-term amplitude modulation (i.e. Blazhko effect) of course remain top priority issues.

\section{Acknowledgements}

The author is grateful for the financial support of the IAU and of the Institute for Fundamental Theory in the Physics Department of the University of Florida. Fruitful discussions with Robert Buchler and Ariel Marom are greatly appreciated. This work was supported by NSF (AST 89-14425) and by an RCI grant through IBM and the NER Data Center at the University of Florida.

\section{References:}

Aikawa, T. and Simon, N. R. 1983, Ap. J., 273, 346.

Antonello, E. and Poretti, E. 1986, Astr. Ap., 169, 149.

Bowen, G. H. 1988, Ap. J., 329, 299.

Buchler, J. R. 1990, in The Numerical Modelling of Nonlinear Stellar Pulsations; Problems and Prospects, Kluwer, Dortrecht; Ed.: J. R. Buchler, p. 1.

Buchler, J. R., Goupil, M.-J. and Kovács, G. 1992, Astr. Ap., submitted.

Buchler, J. R. and Kovács, G. 1992, Physica D, submitted.

Buchler, J. R. and Marom, A. 1992, in preparation.

Buchler, J. R., Moskalik, P., and Kovács, G. 1990, Ap. J., 351, 617.

Buchler, J. R. and Whalen, P. 1990, in The Numerical Modelling of Nonlinear Stellar Pulsations; Problems and Prospects, Kluwer, Dortrecht; Ed.: J. R. Buchler, p. 315.

Castor, J. I., Davis, C. G. and Davison, D. K. 1977, Los Alamos report LA-6664.

Christy, R. 1964, Rev. of Modern Physics, 36, 555.

Davis, C. G. 1972, Ap. J., 172, 419. 
Dorfi, E. A. and Gautschy, A. 1990, in The Numerical Modelling of Nonlinear Stellar Pulsations; Problems and Prospects, Kluwer, Dortrecht; Ed.: J. R. Buchler, p. 289.

Dorfi, E. A. and Feuchtinger, M. U. 1991, Astr. Ap., 249, 417.

Fraley, G. S. 1968, Ap. Space Sci., 2, 96.

Gehmeyr, M. 1991, On Non-Lagrangian Computations of Convective RR Lyrae Stars, $\mathrm{Ph}$. D. Thesis, University of New Mexico.

Goldreich, P. and Keeley, D. A. 1977, Ap. J., 212, 243.

Goldreich, P. and Kumar, P. 1990, Ap. J., 363, 694.

Iglesias, C. A., Rogers, F. J and Wilson, B. G. 1992, Ap. J., submitted.

Kovács, G. 1990, in The Numerical Modelling of Nonlinear Stellar Pulsations; Problems and Prospects, Kluwer, Dortrecht; Ed.: J. R. Buchler, p. 73.

Kovács, G. 1992, in Nonlinear Phenomena in Stellar Variability, IAU Coll. 134, Eds.: J.

R. Buchler, M. Takeuti, in press.

Kovács, G. and Buchler, J. R. 1988, Ap. J., 324, 1026.

Kovács, G. and Buchler, J. R. 1992, Ap. J., in press.

Kovács, G., Buchler, J. R., and Marom, A. 1991, Astr. Ap. (Letters), 252 L27.

Kovács, G., Buchler, J. R., Marom, A., Iglesias, C. A. and Rogers, F. J. 1992, Astr. Ap. (Letters), in press.

Moskalik, P., Buchler, J. R. and Marom, A. 1991, Ap. J., 385, 685.

Rogers, F. J. and Iglesias, C. A. 1992, Ap. J. Suppl., 79, 507.

Simon, N. R. 1982, Ap. J. (Letters), 260, L87.

Simon, N. R. 1985, Ap. J., 299, 723.

Simon, N. R. 1989, Ap. J. (Letters), 343, L17.

Simon, N. R. and Moffett, T. J. 1985, PASP, 97, 1078.

Simon, N. R. and Teays, T. J. 1982, Ap. J., 261, 586.

Stellingwerf, R. F. 1974, Ap. J., 192, 139.

Stellingwerf, R. F. 1975, Ap. J., 195, 441.

Stellingwerf, R. F. 1984, Ap. J., 284, 712.

\section{DISCUSSION}

M. FEUCHTINGER: What kind of rezoning is used in your code?

G. KOVÁCS: The method is very similar to the one what you use, but in the limit of no spatial resolution it goes to a Lagrangean instead of an Eulerian system like in your case.

N. SIMON: To what extent can your new code resolve shocks?

G. KOVÁCS: I let Robert Buchler answer this question.

R. BUCHLER: In principle you can resolve any physical feature, provided you use enough meshpoints. For a given number of meshpoints, however, you have to make a balance between the features you deem most important to be resolved. 